\title{
Spectral and spatial modal control of photonic crystal broad area lasers
}

\section{Lin Zhu, Amnon Yariv}

Lin Zhu, Amnon Yariv, "Spectral and spatial modal control of photonic crystal broad area lasers," Proc. SPIE 7222, Quantum Sensing and Nanophotonic Devices VI, 72220L (26 January 2009); doi: 10.1117/12.814675

Event: SPIE OPTO: Integrated Optoelectronic Devices, 2009, San Jose, California, United States 


\title{
Spectral and spatial modal control of photonic crystal broad area lasers
}

\author{
Lin Zhu*a, and Amnon Yariv ${ }^{\mathrm{b}}$ \\ ${ }^{a}$ Department of Electrical and Computer Engineering and Center for Optical Materials Science and \\ Engineering Technologies, Clemson University, Clemson, SC 29634 \\ ${ }^{\mathrm{b}}$ Department of Applied Physics, California Institute of Technology, Pasadena, CA 91125
}

\begin{abstract}
Photonic crystal structures have been used to improve modal properties of broad area semiconductor lasers in recent years. It has been demonstrated that the width of single-mode semiconductor laser can be increased by at least two orders of magnitude using the transverse Bragg reflection of two dimensional periodic nanostructures. In this talk, we will describe a photonic crystal structure to obtain the single mode operation of large-area, edge-emitting semiconductor lasers. Pulsed and CW operation of electrically pumped, single-mode photonic crystal broad area lasers $(100 \mu \mathrm{m}$ wide and $550 \mu \mathrm{m}$ long) with single-lobe, diffraction-limited far-fields are experimentally demonstrated at room temperature. A wavelength tuning sensitivity 80 times smaller than a conventional DFB laser is also achieved for the photonic crystal Bragg laser.
\end{abstract}

Keywords: modal control, photonic crystal, broad area lasers

\section{INTRODUCTION}

Broad area semiconductor lasers are commercially important especially for high power applications and may find new applications in the integration with other similarly sized components such as microfluidic channels and microelectromechanical systems. These lasers usually have dimensions of about $500 \mu \mathrm{m}$ x $200 \mu \mathrm{m}$ x $100 \mu \mathrm{m}$ (length $\mathrm{x}$ width $\mathrm{x}$ height). They are controlled by electrical current injection and are usually edge-emitting, making them amenable to electronic and optical integration. However, due to their relatively large sizes, broad area lasers usually do not operate in a single mode and their optical characteristics may fluctuate or degrade with current injection. In recent years, photonic crystal structures have been used to overcome the fundamental difficulty of operating broad area (width $>10 \mu \mathrm{m}$ ) semiconductor lasers without filamentation and multi-mode oscillation.

These photonic crystal broad area lasers, also named as two dimensional distributed feedback lasers, select the lasing mode using the Bragg reflection from a two dimensional weak index perturbation. Spatial coherence over a large area can be obtained, allowing for potential single-mode, high power operation with small beam divergence. There are two basic design approaches to two dimensional distributed feedback structures: surface-emitting and edge-emitting [1-7]. For surface-emitting lasers, light has to satisfy the Bragg conditions to diffract out of the plane. Two dimensional distributed feedback surface-emitting lasers have been demonstrated in both semiconductor and polymer materials. Room temperature CW operation has also been obtained for a $50 \mu \mathrm{m} * 50 \mu \mathrm{m}$ single-mode surface-emitting laser [4]. For edge-emitting lasers, the design has to incorporate discrimination mechanisms against gain-guided modes, which prevent the single-mode operation of large-area, edge-emitting semiconductor lasers [8]. Photonic crystal distributed feedback structures with angled facets have been proposed to overcome this problem [6]. Single-mode operation of large-area, edge-emitting photonic crystal semiconductor lasers have been demonstrated in both the pulsed condition $[9,10]$ and $\mathrm{CW}$ condition $[11,12]$.

In this paper, we first introduce the concept of two dimensional distributed feedback structures and explain why these structures can support the single mode operation of a broad area semiconductor laser. We then detail the fabrication process of these photonic crystal broad area lasers in InGaAsP/InP materials. Finally, we show the characterization results of electrically pumped, photonic crystal broad area lasers and discuss some future research directions.

*zhu3@clemson.edu; phone 1864 656-4381; fax 1864 656-5910

Quantum Sensing and Nanophotonic Devices VI, edited by Manijeh Razeghi, Rengarajan Sudharsanan, Gail J. Brown, Proc. of SPIE Vol. 7222, 72220L · C 2009 SPIE · CCC code: 0277-786X/09/\$18 · doi: 10.1117/12.814675 


\section{DESIGN OF PHOTONIC CRYSTAL BROAD AREA LASERS}

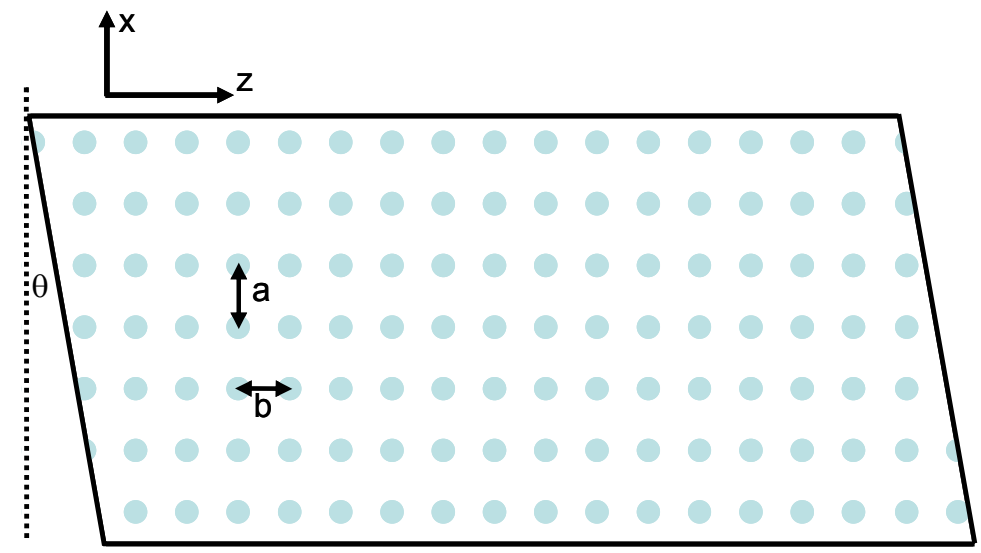

Figure 1: Schematic of a two dimensional photonic crystal broad area laser. $a$ is the transverse lattice constanct, $b$ is the longitudinal lattice constanct, and $\theta_{\text {tilt }}$ is the facet tilt angle.

Figure 1 shows schematic of our photonic crystal laser. The photonic crystal consists of a rectangular lattice array of polymer-filled holes each with a radius of $100 \mathrm{~nm}$ on the wafer surface. The transverse and longitudinal lattice constants are $a$ and $b$, respectively. The active multiple-quantum-well layers are underneath the photonic crystal layer. The cleaved facet is titled relative to the transverse lattice direction with an angle $\theta_{\text {tilt }}$. In the limit of weak index perturbation, which obtains for example for sufficiently small hole diameter, the optical mode for the proposed structure can be separated into transverse (x), vertical (y), and longitudinal (z) components. In the wafer plane (x-z), a mode that satisfies both transverse and longitudinal Bragg resonance conditions will be confined due to the distributed Bragg reflection. Light that does not satisfy the Bragg conditions will be lost. This Bragg condition can be expressed as:

$k_{x}=l \frac{\pi}{a}, k_{z}=j \frac{\pi}{b}(l \neq 0, j \neq 0)$

where $k_{x}$ is the transverse wavevector, $k_{z}$ is the longitudinal wavevector, and $l, j$ are the orders of the grating. Because the vertical wavevector $k_{y}$ is determined by the wafer epitaxial layer structure, $k_{x}$ and $k_{z}$ satisfy:

$k_{x}^{2}+k_{z}^{2}=n_{e f f}^{2} k_{0}^{2}=n_{e f f}^{2}(2 \pi / \lambda)^{2}$

where $n_{\text {eff }}$ is the effective refractive index for the optical mode of the wafer epitaxial structure and $\lambda$ is the resonance (lasing) wavelength. Angled facets are used to suppress effective index-guided modes and gain-guided modes and to ensure the lasing of Bragg-guided modes only. We can define the transverse plane resonance angle $\theta_{\text {res }}$ as: $\theta_{\text {res }}(\lambda)=\cos ^{-1}\left[\sqrt{\left(2 \pi n_{\text {eff }} / \lambda\right)^{2}-(l \pi / a)^{2}} /\left(2 \pi n_{\text {eff }} / \lambda\right)\right]$ and the facet tilt angle $\theta_{\text {tilt }}$ is chosen to be same as the resonance angle $\theta_{\text {res }}$ at $1550 \mathrm{~nm}[13]$. 


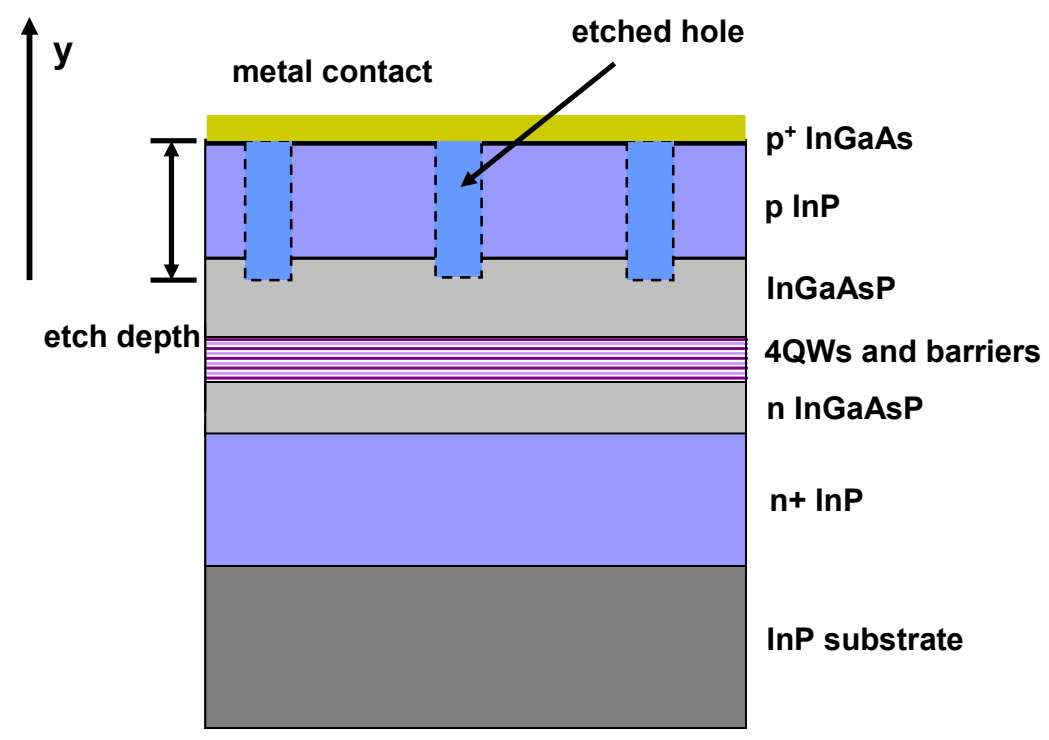

Figure 2: Schematic illustration of the cross section of a photonic crystal broad area laser

Figure 2 shows the schematic illustration of the cross section of a photonic crystal broad area laser. For each etched hole, we usually fill it with the planarization polymer so that the subsequently deposited metal will not fall into the hole, which can cause a lot of absorption loss for the laser. When fabricating the surface photonic crystal, we want to keep the etch depth less than $500 \mathrm{~nm}$ to ensure good etching profiles. Thus we should design the active quantum well region close to the wafer surface. However, since the electrical contact will be directly put on the etched surface, the active region can not be too close to the surface. Otherwise, the metal contact will cause extra absorption losses and lead to a high threshold. With these two constricts, we usually design the active region about $700 \mathrm{~nm}$ away from the wafer surface.

\section{FABRICATION OF PHOTONIC CRYSTAL BROAD AREA LASERS}

The fabrication process can be roughly divided into lithography, etching, planarization, metallization, and packing. First, the photonic crystal pattern is defined by the electron beam lithography. Electron beam lithography is flexible for different designs and provides high resolution features, in comparison to the conventional UV photolithography [48, 49]. In the next step, the photonic crystal is transferred to a dielectric hard mask and then to the semiconductor surface. The etched holes in the semiconductor are then planarized with polymer in order to prevent the metal contact from falling into etched holes. After this, we thermally evaporate electrical contacts on the top and bottom side of the device. Finally, a single device is cleaved and packaged on a C-mount. The whole fabrication process is summarized in Fig. 3.

We start the fabrication by depositing a $120 \mathrm{~nm}$ thick $\mathrm{SiO} 2$ layer on the top of the semiconductor wafer by plasma enhanced chemical vapor deposition (PECVD). A suitable size semiconductor chip is then cleaved from the wafer. Before we spin the ebeam resist for the chip, we need to thoroughly clean the chip using acetone and IPA (isopropanol). Then, a $250 \mathrm{~nm} 495 \mathrm{~K} \mathrm{C} 4$ PMMA (Microchem) resist layer is spun on the substrate at $4000 \mathrm{rpm}$ and the chip is baked on a hotplate at $180^{\circ} \mathrm{C}$ for 3 minutes. We expose the PMMA resist in a Leica Microsystems EBPG 5000+ direct electron beam writer at an accelerating voltage of $100 \mathrm{kV}$ and a beam current of $3.9 \mathrm{nA}$. Development of the patterned PMMA film is carried out in a solution of 1:3 MIBK (methyl-isobutyl ketone) : IPA for 60 seconds. 

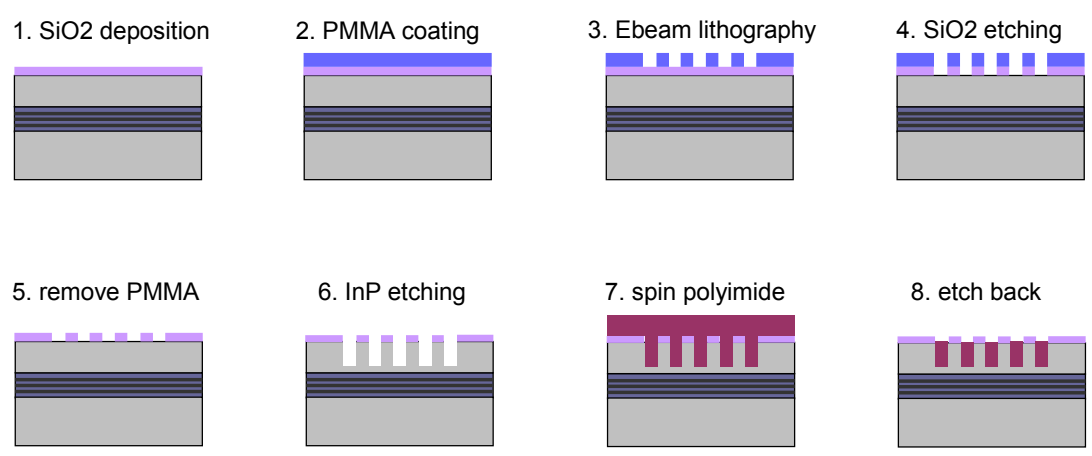

8. etch back
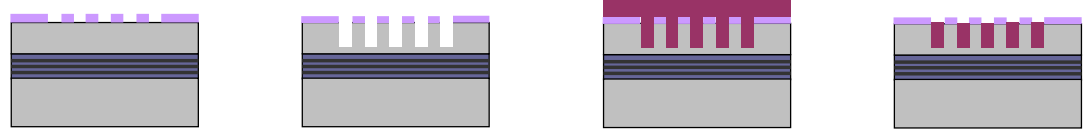

9. remove $\mathrm{SiO} 2$

10. Metallization
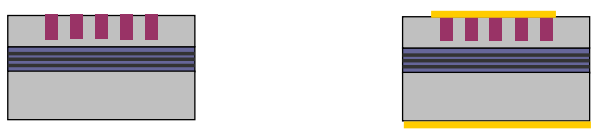

Figure 3: Fabrication process flow

After the PMMA development, the photonic crystal patterns are transferred to the $\mathrm{SiO}_{2}$ layer by reactive ion etching (RIE) using $\mathrm{CHF}_{3}$ plasma. This is because we could not directly use the PMMA layer as a mask to etch InP. The $\mathrm{SiO}_{2}$ layer then serves as a hard mask to etch the semiconductor surface photonic crystal using an ICP-RIE with HI/Ar chemistry. After the etch of InP, the remaining $\mathrm{SiO}_{2}$ layer is about $80 \mathrm{~nm}$ thick. If we remove this leftover silica layer and directly deposit the metal contact at this stage, some of the metal will fall into the etched holes. This part of the metal will be very close to the quantum well region and introduce a lot of absorption losses.

In order to prevent this, we coat the devices with a $2 \mu \mathrm{m}$ thick polyimide (PI-2562, HD Microsystems) planarization layer. The polyimide is subsequently etched back to the $\mathrm{SiO} 2$ layer by an ICP-RIE using pure oxygen. Since the etch depth needs to be accurately controlled, the etch rate should be small. During this process, the sacrificial $\mathrm{SiO}_{2}$ layer provides additional $80 \mathrm{~nm}$ tolerance for the polyimide etch-back process. After the etch-back, we strip off the remaining $\mathrm{SiO}_{2}$ in buffered hydrofluoric (HF) acid solution, creating a polyimide post inside each etched semiconductor hole. In contrast to the regular planarization methods, we can obtain a clean semiconductor surface without any polymer residue due to the $\mathrm{SiO} 2$ isolation layer between the semiconductor and the polymer. Figure 4 shows several SEM images of this planarization process.
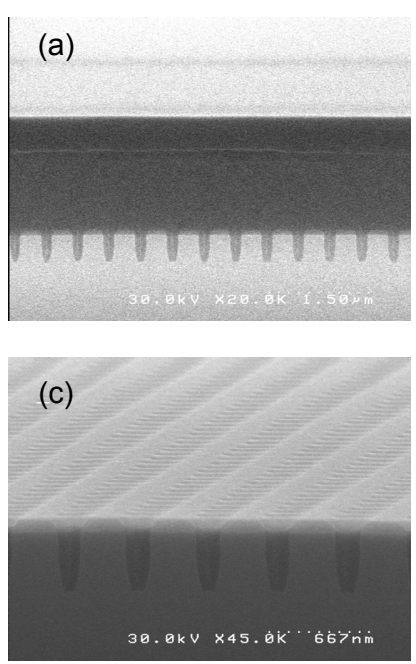
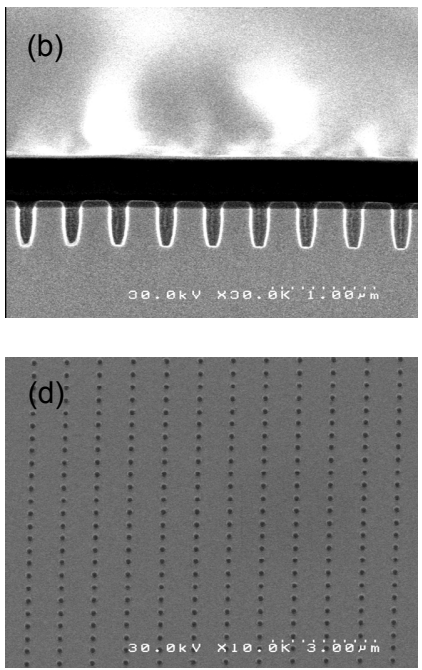

Figure 4: (a) spin on the polyimide (b) etch back the polyimide (c) etch to the $\mathrm{SiO} 2$ layer (d) the planarized sample

Finally, the p-side electrical contact is deposited using a standard lift-off process. A $1.8 \mu \mathrm{m}$ thick layer of 1813 resist (Microposit) is spun on and exposed photolithographically. The p-side contact, $\mathrm{Cr} / \mathrm{AuZn} / \mathrm{Au} 2 \mathrm{~nm} / 6 \mathrm{~nm} / 250 \mathrm{~nm}$, is 
thermally evaporated, covering the photoresist and the areas where the photoresist has been cleared. During the actual lifting-off, the photoresist under the metal layer is removed with acetone, taking the metal with it. The chip is then mechanically thinned to about $100 \mu \mathrm{m}$ thick, and the $\mathrm{n}$-side contact, $\mathrm{Cr} / \mathrm{AuGe} / \mathrm{Au} 2 \mathrm{~nm} / 6 \mathrm{~nm} / 250 \mathrm{~nm}$, is evaporated. The laser bars are then cleaved to lengths of about $500 \mu \mathrm{m}$, and the facets are left uncoated.

\section{CHARACTRIZATION OF PHOTONIC CRYSTAL BROAD AREA LASERS}

\subsection{Room temperature, $\mathrm{CW}$ operation}

We use a first order Bragg reflection for the transverse direction with a lattice constant of $a=1.04 \mu \mathrm{m}$ and a second order Bragg reflection for the longitudinal direction with a lattice constant of $b=490 \mathrm{~nm}$. The design corresponds to a resonance wavelength of $1553 \mathrm{~nm}$ (The effective index $n_{\text {eff }}$ is estimated to be 3.257). The hole radius is $100 \mathrm{~nm}$ and the etch depth is about $400 \mathrm{~nm}$. The metal contact width is $100 \mu \mathrm{m}$ and the tilt angle is $13.8^{\circ}$. The lasers are cleaved to lengths of about $550 \mu \mathrm{m}$ and are p-side up bonded to a C-mount using indium solder. The C-mount is then screwed on a thermoelectric cooling (TEC) stage and the temperature is set at $13^{\circ} \mathrm{C}$.

In order to obtain the room temperature, $\mathrm{CW}$ operation, these lasers are implanted with protons outside the metal contact region. The implantation process can create a high resistance layer in the implanted region. There are two main reasons to do this process: First, since the resistance is higher outside the metal contact stripe, the current will be confined under the metal contact region, preventing the current leakage. In this way, the laser could have a lower threshold. A low threshold is important for the room temperature, CW operation; second, there is no gain outside the electrode due to the confinement of the current. Then gain-guided modes outside the laser will be suppressed. Thus this implantation process can make the performance of the laser more stable with high pump currents.

We measure the light-current (L-I) curve, spectrum, near-field and far-field of the laser. Despite the non-uniform intensity distribution in the near-field, the far-field is single-lobed and diffraction-limited. This proves that the photonic crystal Bragg structure can prevent the filamentation and ensure the single-mode operation.
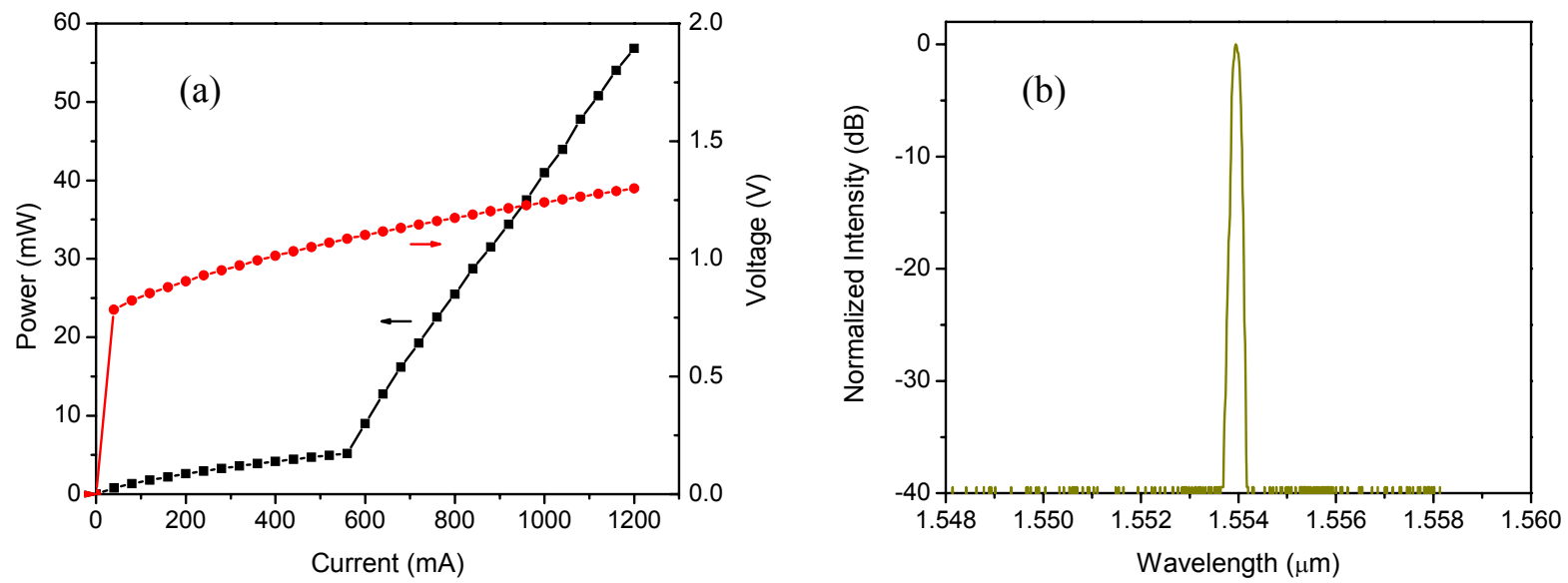

Figure 5: (a) L-I and I-V curves for the photonic crystal Bragg laser. The turn-on voltage is $0.75 \mathrm{~V}$ and the resistance is

$0.68 \Omega$. The threshold is $560 \mathrm{~mA}$. (b) The emission spectrum at the injection current $\mathrm{I}=1.2 \mathrm{~A}$

Figure 5 shows the photonic crystal Bragg laser characteristics of the output power versus the input current (L-I) and the voltage versus the input current (I-V). In the I-V curve, the turn-on voltage of $0.75 \mathrm{~V}$ is much lower compared to our previous devices $(\sim 1.5 \mathrm{~V})$ since we make the planarization polymer slightly higher than the wafer surface. In the L-I curve, the laser has a clear threshold at $560 \mathrm{~mA}$. The device can be operated up to $\sim 2.5 \mathrm{x}$ threshold and further increase of the pumping current leads to thermal rollover. The slope efficiency is $0.08 \mathrm{~W} / \mathrm{A}$, which is similar to the broad area lasers fabricated from the same wafer under the same test conditions. This indicates that this efficiency is mainly limited by the wafer material and the thermal management. Although p-side down bonding provides much better thermal performance, 
we use p-side up bonding since the metal contacts are relatively thin $(\sim 250 \mathrm{~nm})$. Figure $5(\mathrm{~b})$ shows the emission spectrum at an injection current of 1.2A. The single-mode operation is obtained with the side mode suppression ratio (SMSR) higher than $30 \mathrm{~dB}$. The laser also maintains the single-mode operation under different pumping currents.
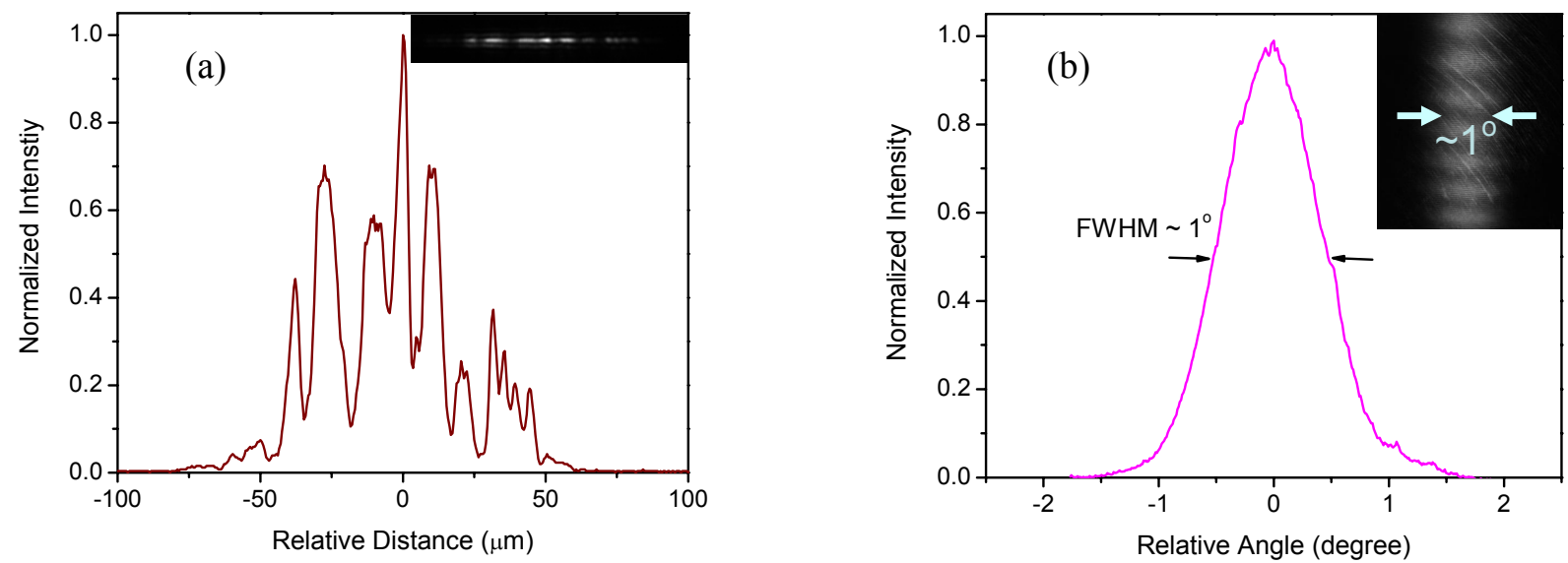

Figure 6: (a) Near field and (b) far field profiles of the photonic crystal Bragg laser (I=1.2A). The insets are direct images captured by infrared CCD cameras

The photonic crystal Bragg laser by limiting operation to a single transverse mode prevents filamentation, which leads to spatial coherence over the large emitting aperture. This results in a single-lobe diffraction-limited far field with small beam divergence. Figure 6 shows the near-field and far-field profiles of the test laser when the pumping current is 1.2A. As shown in Fig. 6(a), the laser has a multi-lobe near-field. This is mainly due to the material gain non-uniformity over a large width $(100 \mu \mathrm{m})$. This kind of non-uniformity generally leads to filamentation for a typical broad area laser or a ridge waveguide laser, resulting in multi-lobe far fields and big beam divergence. However, the far-field of the photonic crystal Bragg laser is single-lobed and possesses a small beam divergence angle of $1^{\circ}$, as shown in Fig. 6(b). (In the CCD far-field image, the fringes in the vertical direction are due to unwanted interferences in the measurement.) The emitting aperture of the laser is about $100 \mathrm{~m}$, corresponding to a theoretical diffraction-limited far-field FWHM (full width at half maximum) width of $0.99^{\circ}$. This shows that the photonic crystal broad area laser can operate in a single-lobe diffraction-limited far-field.

\subsection{Two dimensional wavelength tuning}

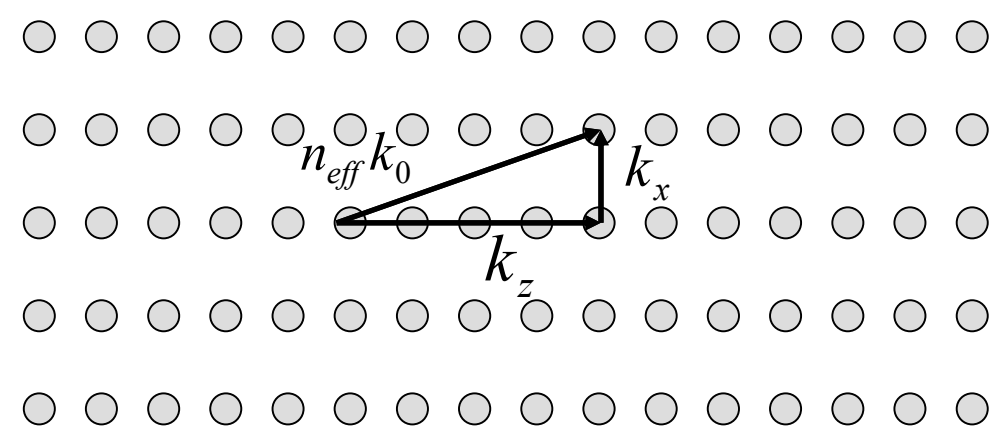

Figure 7: The phase matching conditions for the PC Bragg structure

As shown in Fig. 7, transverse and longitudinal Bragg conditions need to be satisfied simultaneously for the photonic crystal broad area lasers. In another word, we can tune the lasing wavelength by changing either the transverse lattice constant or the longitudinal lattice constant:

$$
\lambda=2 n_{e f f}\left(l^{2} / a^{2}+m^{2} / b^{2}\right)^{-1 / 2}
$$


We then calculate the tuning sensitivity for both the longitudinal and transverse directions from Eq. (3):

$$
\begin{aligned}
& d \lambda / d a=2 n_{\text {eff }}\left(l^{2} / a^{2}+j^{2} / b^{2}\right)^{-3 / 2}\left(l^{2} / a^{3}\right) \\
& d \lambda / d b=2 n_{\text {eff }}\left(l^{2} / a^{2}+j^{2} / b^{2}\right)^{-3 / 2}\left(j^{2} / b^{3}\right)
\end{aligned}
$$

If we design the transverse wavevector much smaller than the longitudinal wavevector, the transverse wavevector only contribute a little to the lasing wavelength. In this way, the lasing wavelength will change a little bit when we change the transverse lattice constant. This means that we can finely tune the lasing wavelength with a relatively large step of the transverse lattice constant.

We first use a first order Bragg reflection $(l=1)$ for the transverse direction with a lattice constant of $a=1 \mu \mathrm{m}$ and a second order Bragg reflection $(j=2)$ for the longitudinal direction with three different lattice constants of $b=480 \mathrm{~nm}$, $490 \mathrm{~nm}$ and $500 \mathrm{~nm}$. With the pulsed current injection, lasing is obtained for the $b=490 \mathrm{~nm}$ and $500 \mathrm{~nm}$ designs with different threshold current densities of $\mathrm{Jth}=1.10 \mathrm{kA} / \mathrm{cm} 2$ and $1.33 \mathrm{KA} / \mathrm{cm} 2$, respectively. Figure 8 (a) shows the optical spectra for all the three designs at the same pump current density $\mathrm{J}=1.40 \mathrm{kA} / \mathrm{cm} 2$. While lasing is not obtained for the device with $b=480 \mathrm{~nm}$, the resonance peak at $1523.1 \mathrm{~nm}$ is evident in the spectrum. The reason that the $b=480 \mathrm{~nm}$ design does not lase is due to the fact that the resonance peak wavelength for this design is far away from the peak wavelength of the gain spectrum and intrinsic losses at short wavelengths are high. Since the resonance wavelength for the $490 \mathrm{~nm}$ design is closer to the gain spectrum peak, the threshold for this design is lower.

In all the three designs, the experimental resonance wavelengths of $1523.1 \mathrm{~nm}, 1550.3 \mathrm{~nm}$, and $1575.9 \mathrm{~nm}$ are close to the theoretical predictions of $1520.2 \mathrm{~nm}, 1550.1 \mathrm{~nm}$ and $1579.9 \mathrm{~nm}$ calculated from Eq. (3). $n_{\text {eff }}$ is chosen to be 3.257 in the calculation and it is numerically calculated by a mode solver at the wavelength of $1550 \mathrm{~nm}$. The slight difference at $1523.1 \mathrm{~nm}$ and $1575.9 \mathrm{~nm}$ is mainly due to the dispersion. Tuning of the longitudinal lattice constant leads to a lasing wavelength tuning sensitivity of 2.6, which is similar to that of regular second order DFB lasers and photonic crystal lasers with large index contrasts.
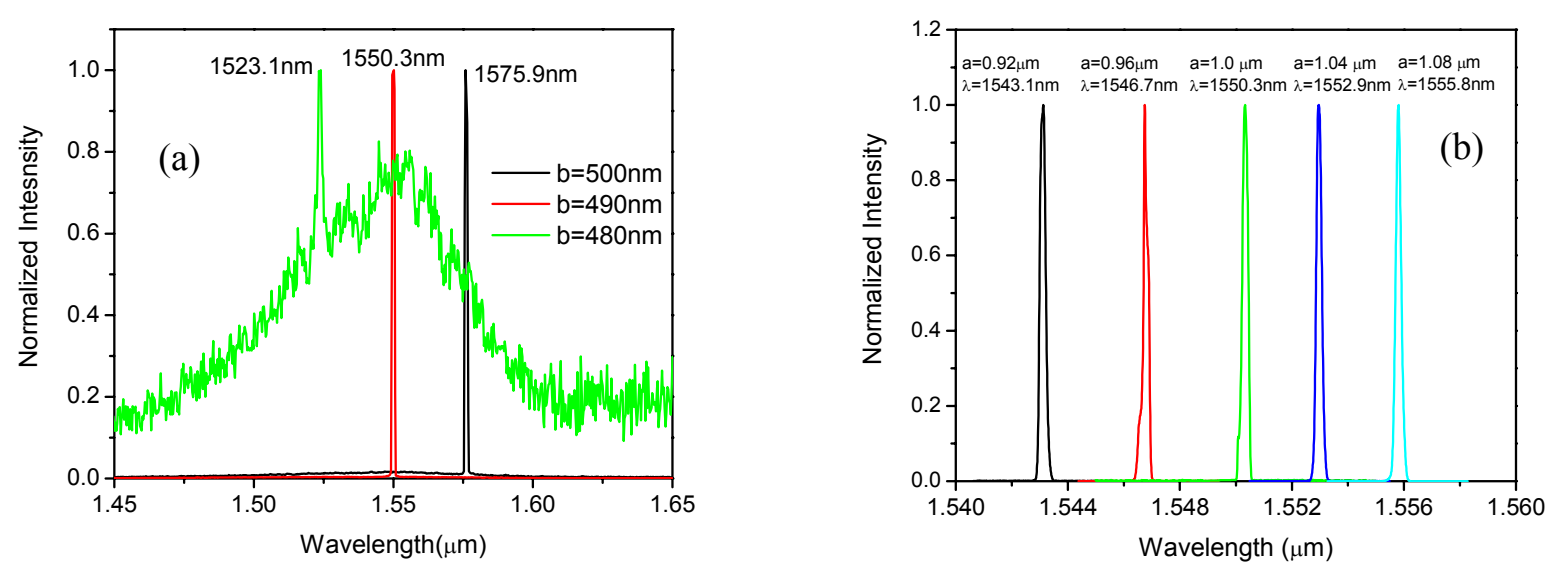

Figure 8: (a) Optical spectra for the lasers with the same transverse lattice constant $\mathrm{a}=1 \mu \mathrm{m}$ but different longitudinal lattice contants $\mathrm{b}=480 \mathrm{~nm}, 490 \mathrm{~nm}$ and $500 \mathrm{~nm}$ at $\mathrm{J}=1.40 \mathrm{kA} / \mathrm{cm} 2$. (b) Lasing spectra for the lasers with the same longitudinal lattice constant $b=490 \mathrm{~nm}$ but different transverse lattice contants $\mathrm{a}=0.92 \mu \mathrm{m}, 0.96 \mu \mathrm{m}, 1.0 \mu \mathrm{m}, 1.04 \mu \mathrm{m}$, and $1.08 \mu \mathrm{m}$ at $\sim 1.5 \times$ threshold

We then fabricate the lasers with the same longitudinal constant of $b=490 \mathrm{~nm}$ and five different transverse lattice constants of $a=0.92 \mu \mathrm{m}, 0.96 \mu \mathrm{m}, 1.0 \mu \mathrm{m}, 1.04 \mu \mathrm{m}$, and $1.08 \mu \mathrm{m}$ on the same chip. Other fabrication parameters are the same as the previous examples. Figure 8 (b) shows the lasing spectra at $\sim 1.5 \times$ threshold for all the five lasers. We also show the lasing wavelength as a function of the transverse lattice constant and corresponding theoretical calculations in Fig. 9. As the transverse lattice constant changes from $0.92 \mu \mathrm{m}$ to $1.08 \mu \mathrm{m}$, the lasing wavelength shifts $12.7 \mathrm{~nm}$. This corresponds to a transverse tuning sensitivity of 0.08 , eighty times smaller than a regular first order DFB laser. The small tuning sensitivity is important for accurate control of the lasing wavelength. If we want the same control accuracy, the fabrication tolerance can be much bigger. 


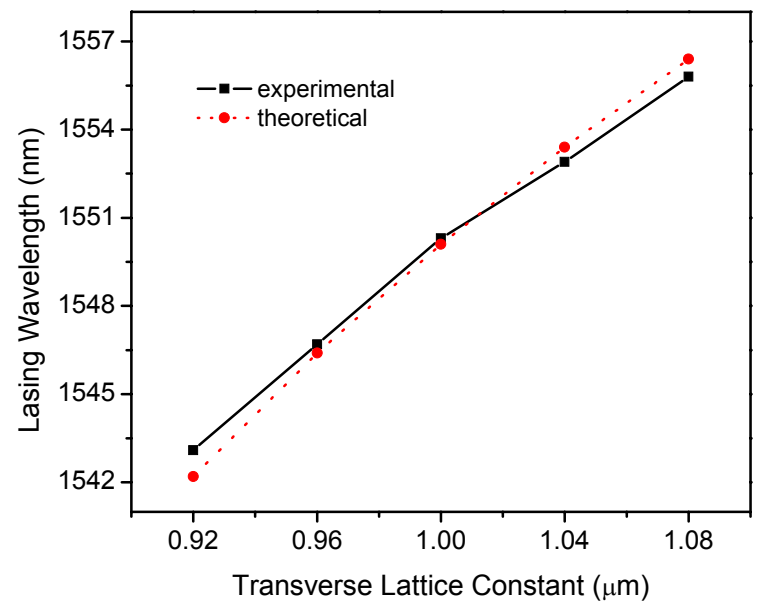

Figure 9: Comparison between the experimental and theoretical results for the lasing wavelength. Good match is obtained and the slight difference is mainly due to the dispersion

\section{DISSCUSSIONS}

We have demonstrated the single mode operation of broad area lasers without multimodes oscillation and filamentation using the surface photonic crystal structure. As we mentioned before, this surface structure requires a short distance between the active region and metal contact, which is not optimal for electrically pumped semiconductor lasers. Also, the surface defects inside the photonic crystal, induced by dry etching during the fabrication process, can cause high threshold and some reliability problems. Thus, a buried photonic crystal is essentially desired and further investigation should focus on the regrowth process of these photonic crystal broad area lasers.

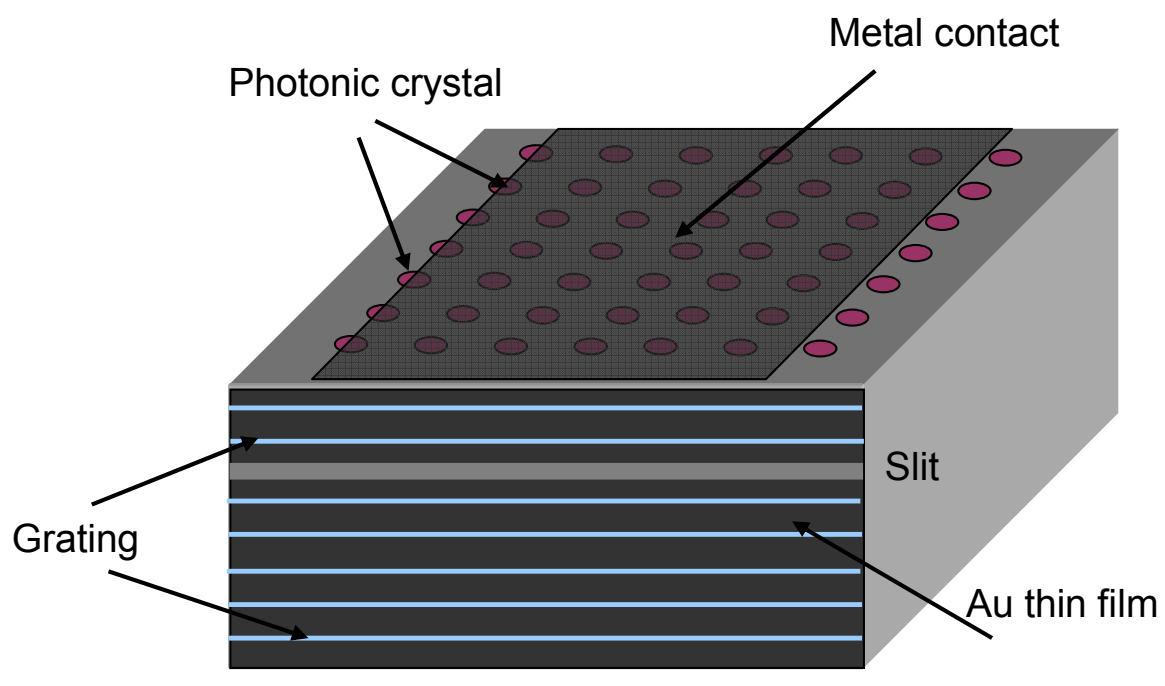

Figure 10: Proposed photonic crystal broad area lasers with integrated plasmonic collimator. There is a dielectric insulation layer between the thin gold film and the laser output facet. This structure can support single mode, high power operation of broad area lasers with highly collimated beams in both vertical and transverse direction in middle infrared and $\mathrm{THz}$ wavelength range. 
Another interesting issue is to narrow down the far field divergence angle in the vertical direction (y direction in Fig. 1 and Fig. 2). Since we just use a standard separate confinement heterostructure (SCH) waveguide design in the vertical direction, the vertical divergence angle is about 30 degree, much bigger than the transverse one (Fig. 6b). To reduce the vertical divergence angle, we can also introduce a Bragg structure in the vertical direction to expand the modal size. However, this is difficult for the fabrication and also leads to a very high threshold. Recently, it has been shown that a small divergence in the vertical direction can be achieved for quantum cascade lasers by defining a metallic subwavelength slit and a grating on their facets [14]. In this design, the slit can efficiently couple the laser output into surface plasmons and the grating can recouple the surface waves into propagating waves. Effectively, the vertical coherent length is expanded so that a small vertical divergence can be obtained. In the visible and near infrared wavelength range, this approach may not be practical due to very high absorption losses. Nonetheless, it is useful in the middle infrared and $\mathrm{THz}$ wavelength range. Integrating this metallic slit-grating structure with our photonic crystal structures, we can achieve single mode, high power operation of broad area lasers with highly collimated beams in both vertical and transverse direction. The proposed structure is shown in Fig. 10.

\section{CONCUSIONS}

This paper reports on achieving the single mode operation of broad area, edge emitting semiconductor lasers, using the photonic crystal structure (two dimensional distributed feedback structure). It is shown that the single mode lasing with a single lobe, diffraction limited far field can be obtained by satisfying both the transverse and longitudinal Bragg conditions of the photonic crystal. Electrically pumped, large-area $(100 \mu \mathrm{m} \times 550 \mu \mathrm{m})$, single mode semiconductor photonic crystal Bragg lasers are experimentally demonstrated in pulsed and continuous wave conditions. Two dimensional lasing wavelength tuning is also obtained, which proves that the lasing mode is truly defined by the photonic crystal lattice. Furthermore, a wavelength tuning sensitivity about 80 times smaller than a conventional DFB laser is also achieved, allowing for more accurate control of the lasing wavelength. Future directions are also discussed, including the regrowth process of the buried photonic crystal structure and the integration of photonic crystal broad area lasers with plasmonic collimators to reduce the vertical beam divergence.

\section{REFERENCES}

[1] S. Wang and S. Sheem, "Two-dimensional distributed-feedback lasers and their applications, "Appl. Phys. Lett. 22, 460-462, 1973

[2] M. Imada, S. Noda, A. Chutinan, T. Tokuda, M. Murata, and G.. Sasaki, "Coherent two dimensional lasing action in surface-emitting laser with triangular lattice photonic crystal structure," Appl. Phys. Lett. 75, 316-318, 1999

[3] S. Noda, M. Yokoyama, M. Imada, A. Chutinan and M. Mochizuki., "Polarization mode control of two-dimensional photonic crystal laser by unit cell structure design," Science 293, 1123-1125, 2001

[4] D. Ohnishi, T. Okano, M. Imada, and S. Noda, "Room temperature continuous wave operation of a surface-emitting two-dimensional photonic crystal diode laser," Opt. Express 12, 1562-1568, 2004

[5] M. Meier, A. Mekis, A. Dodabalapur, A. Timko, R. E. Slusher, J. D. Joannopoulos and O. Nalamasu, "Laser action from two dimensional distributed feedback in photonic crystals," Appl. Phys. Lett. 74, 7-9, 1999

[6] I. Vurgaftman and J.R. Meyer, "Photonic-crystal distributed-feedback lasers," Appl. Phys. Lett. 78, 1475-1477, 2001

[7] C. S. Kim, W. W. Bewley, C. L. Canedy, I. Vurgaftman, M. Kim, and J. R. Meyer, "Broad-stripe near-diffraction-limited mid-infrared laser with a second order photonic crystal distributed feedback grating," IEEE Photon. Technol. Lett.16, $1250-1252,2004$

[8] R.J. Lang, K.D. Zurko, A. Hardy, S. Demars, A. Schoenfelder, and D. Welch. "Theory of grating-confined broadarea lasers," IEEE J. Quantum Electron., 34, 2196-2210, 1998

[9] L. Zhu, G.A. DeRose, A. Scherer, and A. Yariv, "Electrically-pumped, edge-emitting photonic crystal lasers with angled facets," Opt. Lett., 32, 1256-1258, 2007

[10] H. Hofmann, H. Scherer, S. Deubert, M. Kamp, and A. Forchel, "Spectral and spatial single mode emission from a photonic crystal distribution feedback laser," Appl. Phys. Lett., 90, 121135, 2007

[11] L. Zhu, X.K. Sun, G.A. DeRose, A. Scherer, and A. Yariv, "Continuous-wave operation of electrically-pumped, single-mode, edge-emitting photonic crystal Bragg lasers,” Appl. Phys. Lett., 90, 261116, 2007 
[12] K. Paschke, A. Bogatov, F. Bugge, A. E. Drakin, J. Fricke, R. Guther, A. A. Stratonnikov, H. Wenzel, and G. Erbert, "Properties of ion-implanted high-power anged-grating distributed-feedback lasers," IEEE J. Sel. Top Quantum Electron., 9, 1172-1178, 2003

[13] L. Zhu, A. Scherer, and A. Yariv. "Modal gain analysis of transverse Bragg resonance waveguide lasers with and without transverse defects," IEEE J. Quantum Electron., 43, 934-940, 2007

${ }^{[14]}$ N. Yu, J. Fan, Q. J. Wang, C. Pflügl, L. Diehl, T. Edamura, M. Yamanishi, H. Kan, and F. Capasso, "Smalldivergence semiconductor lasers by plasmonic collimation,” Nat. Photonics 2, 564-570, 2008 